\title{
Leishmaniose Visceral em área endêmica do semiárido nordestino: percepção de agentes de saúde e endemias
}

\author{
Visceral Leishmaniasis in endemic area of the semiarid region of brazilian northeastern: \\ perception of community health and combating endemic agents
}

José Gabriel Gonçalves Lins ${ }^{a}$

Orcid: https://orcid.org/0000-0002-0810-1434

Serginara David Rodrigues ${ }^{b}$

Orcid: https://orcid.org/0000-0003-2610-7036

Wennia Motta Galdinoc

Orcid: https://orcid.org/0000-0002-7988-1837
Jânio Pereira Virgíniod

Orcid: https://orcid.org/0000-0002-6117-2119

Thais Ferreira Feitosa ${ }^{\mathrm{e}}$

Orcid: https://orcid.org/0000-0002-2762-6383

Sheila Nogueira Ribeiro Knupp ${ }^{\mathrm{f}}$

Orcid: https://orcid.org/0000-0003-3175-1950

Talles Luann Abrantes Ferreirag

Orcid: https://orcid.org/0000-0002-9702-4388

\begin{abstract}
Resumo
Introdução: A leishmaniose visceral é uma doença parasitária de interesse prioritário, de importância mundial e de caráter zoonótico, que acomete diferentes espécies, dentre elas os cães, gatos, animais silvestres e o homem. A doença tem como vetor o mosquito-palha, do gênero Lutzomyia, da subfamília Phlebotaminae. Objetivo: Objetivou-se avaliar a percepção de agentes comunitários de saúde e de combate às endemias de um município considerado endêmico, diante aos conceitos relacionados à leishmaniose visceral. Materiais e Métodos: O estudo foi desenvolvido entre maio e novembro de 2017, com 60 agentes de saúde e 51 agentes de endemia, selecionados aleatoriamente. Para esses, foi aplicado um questionário estruturado contendo 10 perguntas de múltipla escolha, contendo cinco alternativas em cada pergunta. Após a aplicação dos questionários, foram realizadas palestras e mesas redondas, abordando a epidemiologia, patogenia, profilaxia, controle e tratamento da leishmaniose visceral humana e animal. Resultados: Quanto ao vetor e agente causador, formas de transmissão, de controle e medidas de prevenção da doença, os agentes de combate às endemias apresentaram maior conhecimento comparado aos agentes comunitários de saúde. $83,3 \%$ dos agentes comunitários de saúde e de combate às endemias apresentaram conhecimento considerado bom, frente à cadeia epidemiológica, $\mathrm{e}$ $67,0 \%$ apresentaram conhecimento bom a respeito do tema leishmaniose como zoonose. Conclusões: Os agentes de saúde e de endemias possuem conhecimento acerca do tema,
\end{abstract}

\footnotetext{
a Faculdade de Medicina Veterinária e Zootecnia, Universidade Estadual Paulista, São Paulo, Brasil. E-mail: josegabriel_lins@hotmail.com

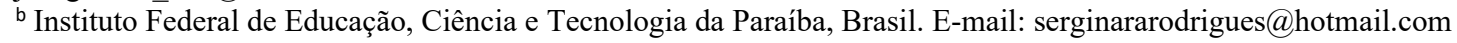

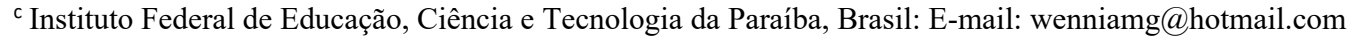

${ }^{d}$ Instituto Federal de Educação, Ciência e Tecnologia da Paraíba, Brasil. E-mail: virginiojanio1@gmail.com

e Instituto Federal de Educação, Ciência e Tecnologia da Paraíba, Brasil. E-mail: feitosa_t@yahoo.com.br

f Instituto Federal de Educação, Ciência e Tecnologia da Paraíba, Brasil: E-mail: sheillanribeiro@hotmail.com

g Instituto Federal de Educação, Ciência e Tecnologia da Paraíba, Brasil. E-mail: talles_abrantesjc@hotmail.com
} 
contudo a realização de cursos de capacitação surge como uma excelente alternativa, para um melhor controle e prevenção da leishmaniose no município estudado.

Palavras-chave: agente comunitário de saúde; agente de combate às endemias; saúde pública; endemicidade.

\begin{abstract}
Introduction: Visceral leishmaniasis (VL) is a parasitic disease of priority interest, especially important worldwide and of zoonotic profile. Such disease affects different species, including dogs, cats, wild animals and humans. The vector mosquito is the phlebotomine sandfly gender Lutzomyia (subfamily Phlebotaminae). Objective: This study aimed to evaluate the perception of community health and combating endemic agents of a municipality considered endemic for VL. Material and Methods: The study was conducted between May and November 2017, involving 60 and 51 community health and combating endemic agents, respectively, randomly selected. It was applied a structured questionnaire based on ten multiple choice questions, each one containing five options. After questionnaires were applied, workshops and round-table meetings were carried out addressing the epidemiology, pathogenesis, prophylaxis, control and treatment of human and animal visceral leishmaniasis Results: Regarding to the vector and disease agent, means of transmission and control, and measures to prevent the disease, the community health agents have presented greater knowledge compared to the combating endemic agents. $83.3 \%$ of the community health and combating endemic agents have shown knowledge described as good in regard to the epidemiological chain. Additionally, $67.0 \%$ of the community health and combating endemic agents have presented knowledge described as good in regard to the leishmaniasis as a zoonosis. Conclusion: The community health and combating endemic agents possess expertise in relation to the disease, however more adequate training courses set up to them, act as an excellent alternative for helping in controlling and preventing leishmaniasis in the study area.
\end{abstract}

Keywords: community health agent; combating endemic agente; public health; endemicity.

\section{Introdução}

As leishmanioses são doenças infecciosas parasitárias, causadas por diferentes espécies de protozoários tripanosomatídeos do gênero Leishmania, transmitidos por flebotamíneos (Diptera, Psychodidae), que acometem principalmente, a pele, mucosas e vísceras de seres humanos, animais silvestres, domésticos e de companhia. Essas doenças são responsáveis por sérios problemas sanitários, socioeconômicos e principalmente, de saúde pública ${ }^{1}$.

Atualmente, a leishmaniose é uma doença de ocorrência não só em ambiente silvestre e rural, mas também de zonas urbanas $^{2}$. A migração da doença, que antes era restrita a ambientes rurais, para os centros urbanos, está relacionada principalmente à adaptação do vetor da doença a locais urbanos com condições de moradia e saneamento básico precários ${ }^{3}$.

Os principais fatores que determinam os altos índices epidemiológicos e urbanização da doença são: o intenso convívio entre o homem e o reservatório do protozoário e a proliferação de insetos vetores, decorrente do desmatamento desordenado ${ }^{4}$. Esses múltiplos fatores ecológicos e epidemiológicos, com as desordens urbanas e outros subjacentes a esse processo, assim como também as dificuldades no acesso e na organização dos serviços de saúde, compõem um cenário 
propício para o desenvolvimento da leishmaniose ${ }^{5}$.

O conhecimento dos dados epidemiológicos de doenças endêmicas tem uma relevante contribuição, quanto à capacidade de alcançar novas estratégias de controle das mesmas ${ }^{6}$. Alguns trabalhos mostram que populações acometidas pela leishmaniose desconhecem conceitos básicos, com relação à transmissão, ao tratamento e à prevenção da doença ${ }^{7}$. Sobretudo, ainda são poucos os estudos na literatura científica que investigam o conhecimento dos agentes comunitários de saúde e de agentes de combate às endemias quanto às leishmanioses.

Os agentes comunitários de saúde são profissionais responsáveis por fortalecer o vínculo entre a equipe multiprofissional e a comunidade, buscando uma melhoria da qualidade de vida das famílias, por meio do desenvolvimento e implementação de ações que visam à prevenção de doenças e promoção da saúde ${ }^{8,9}$.

Os agentes de combate às endemias são profissionais vinculados a uma equipe de vigilância em saúde, responsáveis pela promoção de educação em saúde junto à comunidade, além de informar à população sobre os fatores de risco de doenças, incluindo zoonoses, visando à prevenção e controle dessas. Dessa maneira, a capacitação desses agentes contribui para que esses estejam munidos de conhecimento técnico e científico, tornando-os capazes de identificarem e solucionarem situações de risco, dentre as quais a população pode encontrar-se vulnerável $^{8}$.

Quando avaliada a ocorrência de leishmaniose visceral humana (LVH) no estado da Paraíba (PB), ao longo de oito anos (2010-2017), observou-se que foram notificados 258 casos $^{10}$.

Os casos de leishmaniose visceral humana e leishmaniose visceral canina em
Sousa-PB, entre os anos de 2009 e 2014, foram crescentes, com redução nos anos de 2015 e 2016, mas com retorno do crescimento em $2017^{10}$. No município foram registrados 36 casos de $\mathrm{LVH}$, entre 2009 e $2017^{10,11}$, sendo esses notificados nos bairros Frei Damião, Mutirão, Jardim Sorrilândia I, Jardim Sorrilândia II e Jardim Sorrilândia III ${ }^{11}$. Tais bairros são compostos basicamente por moradias simples, onde a população de menor renda vive em condições precárias de saneamento básico. Regiões periféricas, na maioria das vezes, possuem deficiência na coleta de lixo e saneamento básico, resultando no acúmulo de matéria orgânica, e por consequência, criação de ambientes favoráveis à presença do vetor transmissor da leishmaniose ${ }^{12}$.

Os órgãos de saúde e sistema de vigilância procuram sanar a situação com ações diretas, como por exemplo, a eutanásia de cães diagnosticados positivamente, a fim de possibilitar o controle dessa enfermidade na população, porém a falta de informação limita a adesão da comunidade nesse processo ${ }^{11}$.

Diante do exposto, percebe-se que a situação endêmica do município requer ações preventivas efetivas partindo da equipe de saúde para prevenir o aumento do número de casos da doença. Por isso, objetivou-se investigar a percepção dos agentes comunitários de saúde e dos agentes de combate às endemias do município de Sousa (PB) sobre a leishmaniose visceral.

\section{Metodologia}

O estudo foi desenvolvido entre os meses de maio e novembro de 2017, no município de Sousa (Figura 1), localizado a $438 \mathrm{~km}$ da capital do estado, no Alto Sertão da Paraíba (latitude $6.7625^{\circ} \mathrm{S}$ e longitude $\left.38.2249^{\circ} \mathrm{W}\right)$. A região apresenta clima quente e úmido, com chuvas de verão e temperatura média de $26^{\circ} \mathrm{C}$. 
Figura 1 - Localização geográfica do município de Sousa (Paraíba, Brasil).

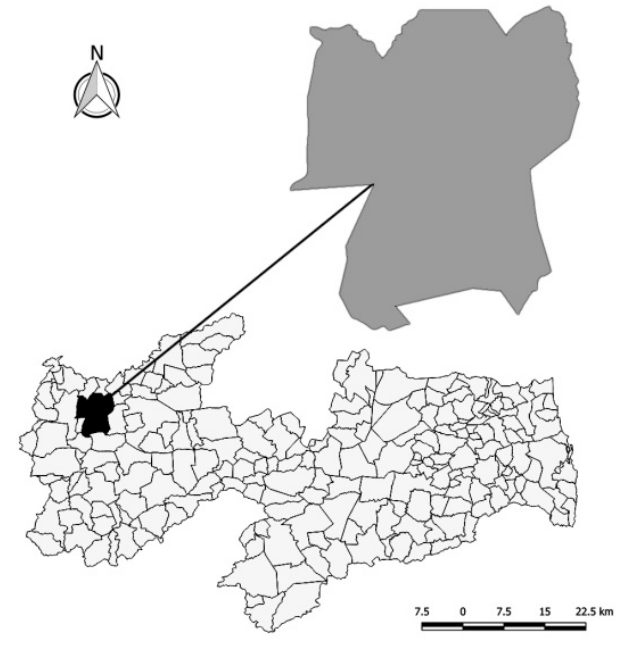

A cidade de Sousa possui uma população de 69.161 habitantes $^{13}$, e está inserida em uma região endêmica para leishmaniose visceral canina, com registros também de casos de leishmaniose visceral humana.

O público alvo do estudo foram os agentes comunitários de saúde (ACS) e os agentes de combate às endemias (ACE) do município, responsáveis pela realização de atividades de prevenção de doenças e promoção da saúde da população.

O município conta com um contingente de 158 ACS e 64 ACE, sendo esses responsáveis pela assistência da população da zona urbana e rural. Para definir o tamanho mínimo da amostra a ser utilizada neste estudo, a amostragem aleatória simples foi calculada ${ }^{14}$ com base no número total de $\mathrm{ACS}$ e $\mathrm{ACE}$ do município. Dessa maneira, foi selecionado um total de 60 agentes comunitários de saúde e 51 agentes de combate às endemias, sendo esses números equivalentes a um intervalo de confiança superior a 95\%.

Foram aplicados questionários estruturados com 10 perguntas de múltipla escolha, contendo cinco alternativas em cada pergunta. As perguntas que compuseram o questionário utilizado foram elaboradas com base no Guia de Vigilância em Saúde - Ministério da Saúde ${ }^{15}$. Anteriormente a aplicação aos ACS e ACE, o questionário foi validado com munícipes de diferentes níveis de escolaridade da cidade de Sousa (PB).

Para a aplicação dos questionários, foram organizados junto à Secretaria Municipal de Saúde, encontros e palestras sobre o tema "Leishmaniose e sua importância na Saúde Pública", entre os meses de agosto e setembro de 2017. Os questionários foram aplicados ao início dos encontros e antes das palestras, sendo os agentes que participaram do estudo, selecionados de forma aleatória. As perguntas e alternativas contidas no questionário seguiram uma mesma ordem para os entrevistados, a fim de assegurar a ausência de variações por parte dos entrevistadores.

Os dados foram tabulados e analisados descritivamente em termos de frequência e valores percentuais. $\mathrm{O}$ percentual médio de acertos foi classificado como: ótimo ( $>90 \%)$, bom $(80-90 \%)$, regular $(50-79 \%)$ e insuficiente $(<50 \%)$. Para a comparação da percepção sobre o tema entre os ACS e ACE, as respostas depois de quantificadas foram submetidas ao teste $t$ pareado por meio do software GraphPad $^{\circledR} \quad 7.0$, sendo os grupos considerados estatisticamente diferentes quando $P<0,05$.

A pesquisa foi aprovada pelo Comitê de Ética e Pesquisa do Instituto Federal de 
Educação, Ciência e Tecnologia da Paraíba (n': 120178/2017).

\section{Resultados e Discussão}

Observou-se que os ACE apresentaram resultados mais satisfatórios comparados aos ACS, quanto ao conhecimento em relação à prevenção e controle da leishmaniose. Os agentes comunitários de saúde apresentaram uma percepção média de $80,83 \pm 5,6 \%$ (percentual médio de acertos \pm erro padrão), enquanto que os agentes de combate às endemias apresentaram uma média de $88,23 \pm 3,5 \%$. Entretanto, quando comparados estatisticamente não foi observada diferença significativa entre as classes $(P=0,0725)$. Os percentuais de acertos obtidos através da aplicação dos questionários estão representados na Figura 2.

Figura 2 - Percepção (\%) dos Agentes Comunitários de Saúde e Agentes de Combate às Endemias do município de Sousa (Paraíba, Brasil) acerca da Leishmaniose Visceral.

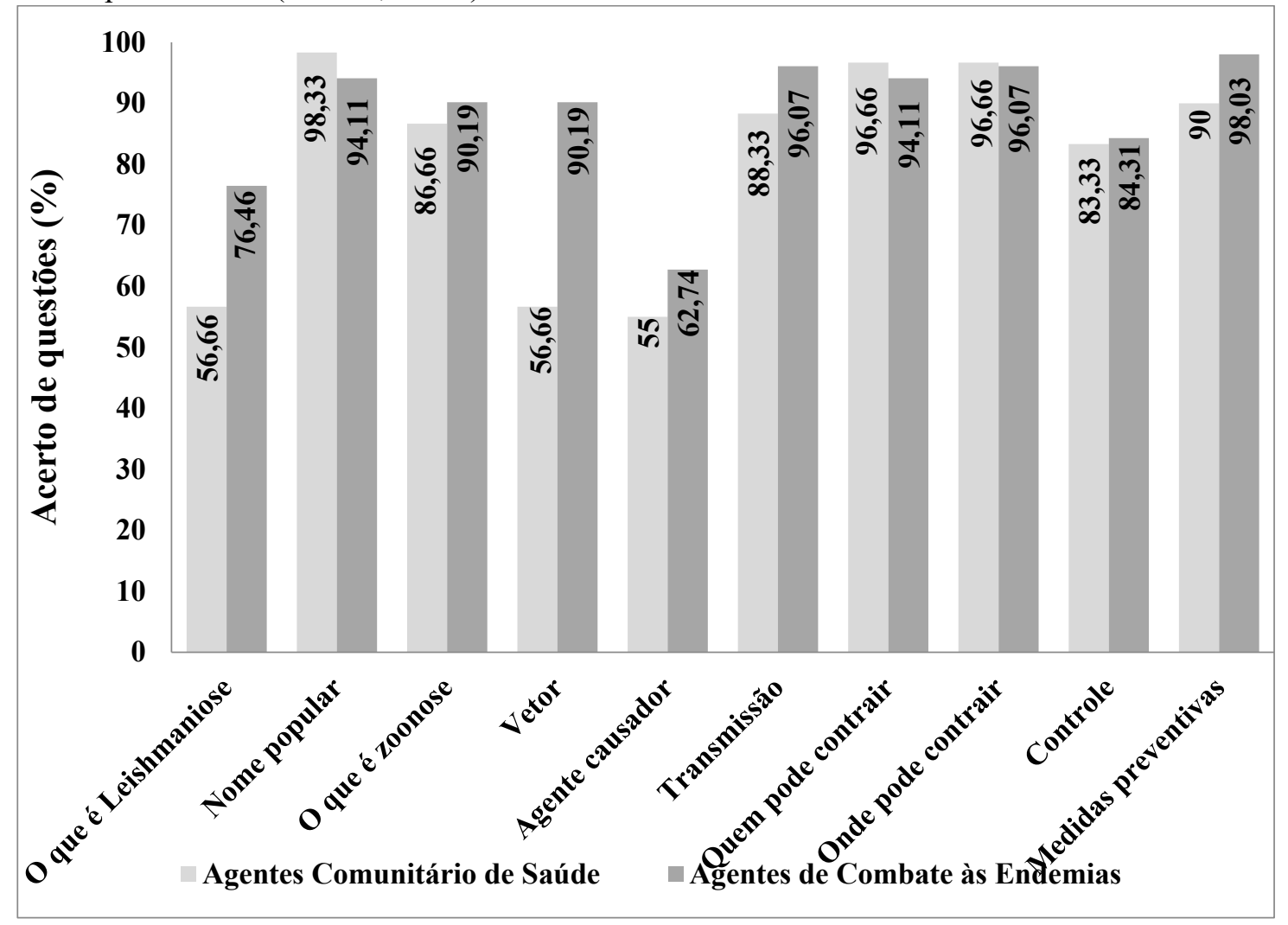

Corroborando com os nossos achados, Carmo et al. ${ }^{5}$ em estudo com profissionais de saúde sobre prevenção e controle da leishmaniose visceral de um município da região metropolitana de Belo Horizonte (MG), os ACE demonstraram maior familiaridade com a doença e com as medidas de prevenção e controle, quando comparados aos ACS. Segundo Costa et al. ${ }^{16}$ em trabalho realizado em Mossoró$\mathrm{RN}$, os ACS apresentaram uma percepção limitada e considerada insuficiente sobre a leishmaniose.
Quando perguntados sobre o que é uma zoonose: 86,66\% (ACS) e 90,19\% (ACE) responderam que zoonose trata-se de uma doença transmitida de animais para humanos; 5,0\% (ACS) disseram tratar-se de uma doença transmitida de humanos para animais; 5,0\% (ACS) e 3,92\% (ACE) afirmaram tratar-se de uma doença transmitida entre animais; $1,66 \%$ (ACS) e $1,96 \%$ (ACE) disseram ser uma doença transmitida entre humanos; $1,66 \%$ (ACS) e $3,92 \%$ (ACE) não souberam responder. Almeida et al. ${ }^{17}$ observaram que $25 \%$ dos 
ACS do município de Quatro Barras (PR) possuíam noções sobre o que são zoonoses. Agentes comunitários de saúde da mesorregião de Araçatuba (SP) ao serem questionados em relação ao significado do termo zoonose, apenas $5.4 \%$ responderam corretamente $^{18}$.

Em relação à caracterização da leishmaniose, 56,66\% (ACS) e 76,4\% (ACE) dos entrevistados a classificaram como uma zoonose importante; 23,33\% (ACS) e $11,76 \%$ (ACE) disseram tratar de uma doença somente de cães; $6,66 \%$ (ACS) e $0,0 \%$ (ACE) disseram ser uma doença de pele causada por sarna; 5,0\% (ACS) e $3,92 \%$ (ACE) afirmaram ser uma doença causada por verme; $3,33 \%$ (ACS) e 3,92\% (ACE) disseram que é uma doença causada por carrapato; $5,0 \%$ (ACS) e 3,92\% (ACE) não souberam responder. Aproximadamente $67,0 \%$ dos ACS e ACE do município de Sousa (PB), apresentaram conhecimento bom a respeito do tema leishmaniose como zoonose, sendo esse percentual superior ao encontrado por Costa et al. ${ }^{16}$, em que aproximadamente $26 \%$ dos ACS da região metropolitana de Recife (PE) apresentaram um conhecimento considerado bom quanto à leishmaniose como zoonose, enquanto que os $\mathrm{ACE}$ apresentaram um percentual de $28,9 \%$.

Quanto ao nome popular da doença leishmaniose: 98,33\% (ACS) e 94,11\% (ACE) afirmaram conhecer a doença como "calazar"; 1,66\% (ACS) relataram conhecer a doença como sarna; e 3,92\% (ACE) disseram conhecer como caxumba; e 1,96\% (ACE) afirmaram conhecer a doença como raiva. Menezes et al. ${ }^{7}$ observaram que $70 \%$ dos ACS conheciam o "calazar" como nome popular da Leishmaniose Visceral, enquanto que $13,3 \%$ afirmaram ser a enfermidade conhecida com leptospirose.

Segundo Costa et al. ${ }^{16}$ quando os ACS e ACE foram perguntados sobre a cadeia epidemiológica da doença, $62,8 \%$ apresentaram conhecimento regular, $28,6 \%$ um bom conhecimento e $8,6 \%$ mostraram conhecimento insuficiente. $\mathrm{O}$ mesmo não aconteceu para os agentes da cidade de
Sousa (PB), onde se pôde observar que aproximadamente $83,3 \%$ dos ACS e ACE apresentaram conhecimento considerado bom frente à cadeia epidemiológica.

Em relação ao conhecimento sobre o vetor da doença: $56,7 \%$ (ACS) e 90,2\% (ACE) disseram ser o mosquito palha o transmissor da leishmaniose; $28,3 \%$ (ACS) e 9,8\% (ACE) afirmaram ser os cães; e $15,0 \%$ (ACS) disseram ser o carrapato. Massia $^{19}$ observou que $100 \%$ dos ACE e $86 \%$ ACS do município de Uruguaiana (RS) afirmaram que a transmissão da leishmaniose acontece por meio da picada do mosquito palha. Costa et al. ${ }^{20}$ observaram que aproximadamente $30 \%$ desses profissionais indicaram o cão como transmissor da leishmaniose. Menezes et al. ${ }^{7}$ observaram que $66 \%$ dos ACS possuíam noções sobre a transmissão da Leishmaniose Visceral, mas $8.4 \%$ afirmaram que a doença é transmitida pela mordida de cães infectados, e 10.9\% indicaram que a transmissão ocorre pela picada do Aedes aegypti infectado.

Quanto ao agente causador da doença: $55,0 \% \quad(\mathrm{ACS}) \quad$ e $\quad 62,74 \% \quad$ (ACE) responderam ser um protozoário; $15,0 \%$ (ACS) um ácaro; 11,66\% (ACS) e 21,96\% (ACE) um vírus; 8,33\% (ACS) e 11,76\% (ACE) bactéria; e 10,0\% (ACS) e 3,92\% (ACE) não souberam responder.

Quando questionados sobre a transmissão: 88,33\% (ACS) e 96,07\% (ACE) responderam que a doença é transmitida por meio da picada do mosquito; 5,0\% (ACS) e 1,96\% (ACE) disseram ser através da ingestão de alimentos contaminados; 3,33\% (ACS) e $1,96 \%$ (ACE) responderam ser por intermédio do contato direto com o sangue de animais contaminados; $1,66 \%$ (ACS) que a doença pode ser contraída através da mordida do cão doente; e 1,66\% (ACS) não souberam responder. Em estudo desenvolvido por Torres ${ }^{18}, 10,8 \%$ dos ACS afirmaram erroneamente que o cão poderia transmitir a doença para as pessoas sem a presença do vetor, por meio do contato direto com o animal, fezes e urina, assim 
como também pela mordida, e 7,52\% afirmaram que o vetor da leishmaniose visceral é o Aedes aegypti.

Sobre quem pode contrair: $96,66 \%$ (ACS) e 94,11\% (ACE) responderam corretamente que humanos, cães, raposas e cachorros de mato, gambás, equídeos, roedores e gatos podem adquirir a doença; $1,96 \%$ (ACE) responderam que apenas cães, aves, coelhos e anfíbios podem contrair; 1,96\% (ACE) que apenas galinhas, raposas, morcegos e peixes contraem a doença; 1,66\% (ACS) afirmaram que apenas humanos, corujas, cães, peixes e anfíbios podem contrair leishmaniose; e $1,66 \%$ (ACS) e 1,96\% (ACE) não souberam responder. Ainda, quanto ao local onde se pode contrair a doença: $96,66 \%$ (ACS) e 96,07\% (ACE) responderam que a doença pode ser contraída tanto em ambiente rural, como em ambiente urbano. 1,96\% (ACE) responderam que a doença pode ser contraída apenas na zona rural; 1,66\% (ACS) apenas na zona urbana; e 1,66\% (ACS) e 1,96\% (ACE) não souberam responder. Torres ${ }^{18}$ observou que $9,67 \%$ dos ACS citaram erroneamente que, além do cão, bovinos e galinhas podem adquirir leishmaniose visceral.

Em relação à forma de controle da leishmaniose: 83,33\% (ACS) e 84,31\% (ACE) responderam que a forma de combater a doença é prevenir a disseminação do mosquito, informar a população sobre os riscos, diagnosticar os portadores, sacrificar os animais acometidos e tratar adequadamente os humanos com essa doença; 11,66\% (ACS) e 9,8\% (ACE) disseram que pode combater a doença, passeando com o cão, principalmente, no final da tarde e início da manhã; 1,66\% (ACS) e 1,96\% (ACE) responderam que a melhor forma de controle é sacrificar todos os animais abandonados (doentes e saudáveis); 1,66\% (ACS) e 3,96\% (ACE) afirmaram que com o desmatamento, evita-se a presença do mosquito nas proximidades domiciliares; e $1,66 \%$ (ACS) não souberam responder. Menezes et al. ${ }^{7}$ observaram que $79,5 \%$ dos
ACS indicaram adequadamente as formas de controle da enfermidade.

Massia $^{19}$ identificou que $82 \%$ dos ACE e $41 \%$ dos ACS informaram que eliminar os cães infectados seria a melhor alternativa, mas também responderam que o tratamento de cães infectados $(18 \%$ dos ACE e $50 \%$ dos ACS) é uma medida que deveria ser adotada. De acordo com o Ministério da Saúde, dentre as medidas de controle da leishmaniose visceral em cães, são recomendados o diagnóstico precoce e a eutanásia de cães soropositivos ${ }^{21}$.

Por fim, quanto à maneira de proteger-se da doença: 90,0\% (ACS) e $98,03 \%$ (ACE) disseram que é preciso colocar telas nas janelas, usar repelente e realizar a limpeza do ambiente; 3,33\% (ACS) e 1,96\% (ACE) responderam que deve-se abrir as janelas no final da tarde e início da manhã, para que os mosquitos possam sair; 3,33\% (ACS) disseram que deve-se abandonar os cães nas ruas para que a doença seja mantida longe de casa; e $3,33 \%$ (ACS) não souberam responder. Massia $^{19}$ observou que $100 \%$ dos ACE e 94\% dos ACS responderam que manter o pátio limpo e sem matéria orgânica seriam as melhores medidas preventivas da leishmaniose visceral. Ainda, $17 \%$ dos ACS responderam ainda que eliminar água parada é uma excelente medida preventiva. Menezes $^{7}$ identificou que $64 \%$ dos ACS conheciam as medidas de prevenção da doença, mas observou que $27,7 \%$ responderam que cobrir as caixas d'água e não deixar águas empoçadas eram excelentes medidas preventivas.

Apesar de estatisticamente não observarmos diferenças significativas, foi notado um conhecimento regular dos ACS $(<60,0 \%)$ quanto à definição, vetor e agente causador da doença, mesmo sendo essa uma doença endêmica na cidade em que atuam. Os ACE mostraram-se mais familiarizados com o tema, apresentando um percentual médio de acerto superior a $88,0 \%$. Tal diferença entre a percepção dos dois diferentes grupos de profissionais pode ser 
atribuída, principalmente, às diferentes atribuições desses profissionais.

Werneck et al. ${ }^{22}$ apresentam a leishmaniose como uma doença, na maioria das vezes, de baixa prioridade por parte das políticas e serviços de saúde. Isso reflete em uma baixa oferta de atividades de atualização contínua das práticas em saúde sobre LV para os $\mathrm{ACS}^{20}$. Os profissionais de saúde pública ainda possuem dúvidas a respeito da leishmaniose visceral, bem como demandam capacitações relacionadas ao tema ${ }^{5}$. Contudo, a atuação conjunta dos profissionais de assistência comunitária pode facilitar a disseminação do conhecimento e, progressivamente, efetivar o ciclo informativo e preventivo sobre as enfermidades, como as leishmanioses por exemplo ${ }^{7}$.

Após a aplicação dos questionários foram realizadas palestras e mesas redondas, e foram abordados os principais conceitos acerca da epidemiologia, profilaxia e controle da leishmaniose visceral. Os agentes que participaram dos encontros aproveitaram a oportunidade para enfatizar a importância da capacitação profissional e do desenvolvimento de ações conjuntas entre os profissionais da saúde no controle de várias doenças. Os ACS e ACE são os atores mais próximos da população, sendo suas ações essenciais na divulgação de informações e detecção de casos suspeitos.

\section{Conclusão}

A percepção geral acerca da leishmaniose visceral por parte dos agentes comunitários de saúde e dos agentes de combate às endemias foi considerada boa, mas ainda existem algumas lacunas em aspectos conceituais. O presente estudo reforçou a importância acerca da necessidade de desenvolver ações que visem manter, atualizar e expandir $o$ conhecimento desses profissionais por meio de programas de educação continuada contextualizada à realidade do município, contribuindo assim para uma diminuição da casuística de doença no município de Sousa $(\mathrm{PB})$ e região.

Os agentes comunitários de saúde e de combate às endemias podem funcionar como excelente ferramenta de combate à doença dentro do município, através do esclarecimento e inclusão da população, para que ela contribua de forma efetiva no controle e prevenção da doença. Uma limitação deste estudo foi o não acompanhamento em longo prazo desses profissionais após a realização das palestras e mesas redondas, para uma avaliação a respeito da aquisição de conhecimento sobre a Leishmaniose Visceral.

\section{Agradecimentos}

Agradecemos a Lucas Queiroga de Sousa e Welitania Inácia da Silva pelo apoio técnico, à Secretaria Municipal de Saúde de Sousa/PB pela articulação e mobilização das ações, e ao Instituto Federal de Educação, Ciência e Tecnologia da Paraíba campus Sousa, pelo apoio financeiro.

\section{Referências}

1. Boraschi CSS, Nunes CM. Aspectos epidemiológicos da leishmaniose visceral urbana no Brasil. Clínica Veterinária 2007; 71: 44-48.

2. Gontijo CMF, Melo MN. Leishmaniose Visceral no Brasil: quadro atual, desafios e perspectivas. Rev Bras Epidemiol 2004; 7(3): 338-349.

3. BRASIL. Ministério da Saúde (MS). Secretaria de Vigilância em Saúde. Departamento de Vigilância Epidemiológica. Manual de Vigilância e Controle da Leishmaniose Visceral. Brasília: MS; 2006. 
4. Boraschi CSS, Perri SHV, Nunes CM. Leishmaniose visceral: o que a população de Três Lagoas, MS, Brasil, sabe sobre esta enfermidade? Vet e Zootec 2008; 15(3): 478-485.

5. Carmo RF, Luz ZMP, Bevilacqua PD. Percepções da população e de profisssionais de saúde sobre a leishmaniose visceral. Cien Saude Colet 2016; 21(2): 621-628.

6. Gama MEA, Barbosa JS, Pires B, Cunha AKB, Freitas AR, Ribeiro IR, Costa JML. Avaliação do nível de conhecimento que populações residentes em áreas endêmicas têm sobre leishmaniose visceral, Estado do Maranhão, Brasil. Cad Saúde Pública 1998; 14(2):.381-390.

7. Menezes JÁ, Luz TCB, Sousa FF, Verne RN, Lima FP, Margorani C. Fatores de risco peridomiciliares e conhecimento sobre leishmaniose visceral da população de Formiga, Minas Gerais. Rev Bras Epidemiol 2016; 19(2): 362-374.

8. Pereira HS, Limongi JE. Agentes comunitários de saúde: atribuições e desafios. Hygea 2011; 7(12): 83-89.

9. Knochenhauer CCL, Vianna KMP. Percepção dos Agentes Comunitários de Saúde quanto aos agravos fonoaudiológicos. CoDAS 2016; 28(6): 697-703.

10. BRASIL. Ministério da Saúde (MS). Secretaria de Vigilância em Saúde. Departamento de Vigilância Epidemiológica. Sistema de Informação de Agravos de Notificação. [acesso 05 jul 2018]. Disponível em: http://www2.datasus.gov.br

11. Lins JGG, Andrade RCS, Arruda LFB. Fonseca Júnior AM, Sousa MS, Clementino WKL, Evangelista IL. Percepção sobre a doença leishmaniose em área endêmica no município do alto sertão paraibano. Anais do II Encontro de Inciação Científica e I Encontro de Pesquisadores do IFPB Campus Sousa, 2012 Dez 14, Sousa, Brasil. Sousa: IFPB; 2012.

12. VIANA, A. G., et al. Aspectos clínico-epidemiológicos da leishmaniose tegumentar americana em Montes Claros, Minas Gerais. Rev Med Minas Gerais, 2012.

13. Instituto Brasileiro de Geografia e Estatística (IBGE). Projeções da População. Rio de Janeiro: IBGE; 2018.

14. OpenEpi [homepage na internet]. Toolkit Shell for Developing New Applications. [Acesso 23 jun 2017]. Disponível em: http://www.openepi.com/SampleSize/SSPropor.htm

15. Brasil. Ministério da Saúde. Secretaria de Vigilância em Saúde. Coordenação Geral de Desenvolvimento da Epidemiologia em Serviços. Guia de Vigilância em Saúde. 1. ed. Brasília: Ministério da Saúde, 2016. [Acesso 08 abr 2018] Disponível em: http://portalarquivos2.saude.gov.br/images/pdf/2016/novembro/18/Guia-LV-2016.pdf.

16. Costa GJA, Cavalcanti SMA, Lins Filho NM, Della Bella S, Marvulo MFV, Bezerra R, Alves LC, Silva JCR. Avaliação da percepção sobre zoonoses com agentes de saúde, combate a endemias e docentes de escola públicas, do entorno da Estação Ecológica de Caetés, Região Metropolitana do Recife-PE, Brasil. Medicina Veterinária (UFRPE) 2017; 11(1): 67-75.

17. Almeida, JC, Bollmann C, Marinho AP, Skalski J, Amaral CH, Biondo AW, Svoboda WK, Wouk AFPF. Percepção sobre zoonoses de professores e Agentes Comunitários de Saúde do Município de Quatro Barras - PR. Higiene Alimentar, 23: 602-603, 2009.

18. Torres APC. Programa de orientação para controle de Leishmaniose Visceral canina. 2017. 40f. Tese (Doutorado em Medicina Veterinária). Universidade Estadual Paulista, Jaboticabal.

19. Massia LI. Leishmaniose visceral: avaliação do conhecimento dos agentes de saúde pública em Uruguaiana (RS). 2017. 85f. Dissertação (Mestrado em Ciência Animal). Universidade Federal do Pampa, Uruguaiana.

20. Costa KFL. Percepção e diagnóstico da Leishmaniose visceral canina em áreas ribeirinhas na cidade de Mossoró, Rio Grande do Norte. 2014. 93f. Dissertação (Mestrado em Ambiente, Tecnologia e Sociedade). Universidade Federal Rural do Semiárido, Mossoró.

21. BRASIL. Ministério da Saúde. Departamento de Vigilância Epidemiológica. Manual de vigilância e controle da leishmaniose visceral. 1. ed., 5. reimpr. Brasília: Ministério da Saúde, 2014. 
22. Werneck GL, Hasselmann MH, Gouvêa TG. Panorama dos estudos sobre nutrição e doenças negligenciadas no Brasil. Ciênc saúde coletiva. 2011; 16(1):39-62.

\section{Como citar este artigo:}

Lins JGG, Rodrigues SD, Galdino WM, Ferreira TLA, Virgínio JP, Feitosa TF, Knupp SNR. Leishmaniose Visceral em área endêmica do semiárido nordestino: percepção de agentes de saúde e endemias. Rev. Aten. Saúde. 2020; 18(64): 32-41. 